\title{
A Uniqueness Theorem for Initial-value Problems
}

\author{
By
}

\author{
Masatake MryAKE*
}

\section{Introduction}

We shall consider in this note the following linear partial differential operator,

$$
P(x, \partial / \partial x)=\sum_{i+j \leqq m} a_{i j}(x) \partial^{i+j} / \partial x_{1}^{i} \partial x_{2}^{j},
$$

where $a_{i j}(x)$ are analytic functions defined in an open set $\Omega \subset R^{2}$, and of real-valued if $i+j=m$.

Let $\mathscr{S}$ be an analytic curve defined by $\varphi(x)=\varphi\left(x^{0}\right), x^{0} \in \Omega$, where $\varphi(x)$ is a real-valued analytic function defined in $\Omega$. From now on we assume that $\varphi_{x_{1}}\left(x^{0}\right) \neq 0$.

Now let us assume that $\mathscr{S}$ is a double characteristic curve of (1), that is,

$$
\begin{aligned}
& \left.P_{m}\left(x, \varphi_{x}\right)\right|_{\mathscr{S}}=0,\left.\quad P_{m}^{(i)}\left(x, \varphi_{x}\right)\right|_{\mathscr{S}}=0 \quad \text { for } i=1,2, \\
& \left.P_{m}^{(i, j)}\left(x, \varphi_{x}\right)\right|_{\mathscr{S}} \neq 0 \quad \text { for some } i, j=1,2,
\end{aligned}
$$

where $P_{m}(x, \xi)=\sum_{i+j=m} a_{i j}(x) \xi_{1}^{i} \xi_{2}^{j}, P_{m}^{(i)}(x, \xi)=\partial P_{m}(x, \xi) / \partial \xi_{i}$ and $P_{m}^{(i, j)}(x$, $\xi)=\partial^{2} P_{m}(x, \xi) / \partial \xi_{i} \partial \xi_{j}$.

And also we assume that $\boldsymbol{P}_{m}\left(x, \varphi_{x}\right)$ vanishes at the first order on $\mathscr{S}$, that is,

$$
\left.<\varphi_{x}, \partial / \partial x>\left.P_{m}\left(x, \varphi_{x}\right)\right|_{\mathscr{S}} \neq 0, \quad<\varphi_{x}, \partial / \partial x\right\rangle=\sum_{i=1}^{2} \varphi_{x_{i}} \frac{\partial}{\partial x_{i}}
$$

Received September 21, 1972.

Communicated by S. Matsuura.

* Department of Mathematics, Faculty of Science, Kanazawa University, Kanazawa, Japan. 
Recently, Y. Hasegawa [3], [4] has proved the existence theorem of the initial-value problem with data on a characteristic surface, and the system case was treated by the author [8]. In those papers, the initialvalue problems were classified in many cases using the lower order terms of the differential operator $P(x, \partial / \partial x)$. One of the purposes of this note is to investigate a geometrical meaning of the double characteristic curve under the assumption (3) which did not appear in J. Vaillant [10] but appeared in Y. Hasegawa [3] and the author [8] (see Proposition in §1).

On the other hand, L. Hörmander $[5],[6], F$. Trèves [9] and E. C. Zachmanoglou [11], [12] proved uniqueness theorems of the initialvalue problems when the initial surface has simple characteristic points under a convexity condition or modified conditions. And also, J.M. Bony [2] and L. Hörmander [7] proved uniqueness theorems which are extensions of Holmgren's theorem. Another purpose of this note is to show a uniqueness theorem of the distribution solution of $P(x, \partial / \partial x) u=0$ when the initial curve has double characteristic points (see Theorem in $\S 1$ ).

Finally, the author wishes his sincere gratitude to Prof. S. Mizohata for his valuable suggestion and encouragement.

\section{$\S 1$. Statement of Theorem}

At first we note that we have $P_{m}^{(2,2)}\left(x^{0}, \varphi_{x}\left(x^{0}\right)\right) \neq 0$ from the condition (2) and $\varphi_{x_{1}}\left(x^{0}\right) \neq 0$. And also we have

$$
\operatorname{sgn}\left[P_{m}^{(2,2)}\left(x^{0}, \varphi_{x}\left(x^{0}\right)\right)\right]=\operatorname{sgn}\left[\sum_{i, j} P_{m}^{(i, j)}\left(x^{0}, \varphi_{x}\left(x^{0}\right)\right) \psi_{x_{i}}\left(x^{0}\right) \psi_{x_{j}}\left(x^{0}\right)\right],
$$

where $\phi(x)$ is a real-valued analytic function defined in $\Omega$ such that $\partial(\varphi$, $\psi) /\left.\partial\left(x_{1}, x_{2}\right)\right|_{x=x^{0}} \neq 0$.

Without loss of generality, we may assume that $\operatorname{sgn}\left[<\varphi_{x}, \frac{\partial}{\partial x}>P_{m}(x\right.$, $\left.\left.\varphi_{x}\right)\left.\right|_{x=x^{0}}\right]$ and $\operatorname{sgn}\left[P_{m}^{(2,2)}\left(x^{0}, \varphi_{x}\left(x^{0}\right)\right)\right]$ are different. In fact, if they are the same, we then consider $-\varphi(x)$ instead of $\varphi(x)$. Then we have the following

Proposition. Under the assumptions (2), (3) and the above, there exist two and only two analytic characteristic curves through each point in 
$\varphi(x)>\varphi\left(x^{0}\right)$ and sufficiently near $\mathscr{S}$ such that they are tangent at the second order to $\mathscr{S}$. And the other characteristic curves are transversal to $\mathscr{S}$.

From this proposition we may consider that $\mathscr{S}$ is an envelope of characteristic curves of (1). We remark that if we assume that $<\varphi_{x}$, $\frac{\partial}{\partial x}>\left.P_{m}\left(x, \varphi_{x}\right)\right|_{\mathscr{S}}=0$ instead of the assumption (3), we can not obtain a similar result as in the Proposition.

Let $\mathscr{C}$ be a regular curve defined by $f(x)=f\left(x^{0}\right)$, tangent at $x^{0}$ to the double characteristic curve $\mathscr{S}$, where $f(x)$ is a real-valued function of $C^{1}(\Omega)$, and we may assume

$$
f_{x}\left(x^{0}\right)=\varphi_{x}\left(x^{0}\right)
$$

Now we impose on $\mathscr{C}$ the following conditions:

$$
\begin{aligned}
& \text { If } x_{2}>x_{2}^{0}\left(\text { or } x_{2}<x_{2}^{0}\right), f(x)<f\left(x^{0}\right) \text { on } \mathscr{S} \text {, } \\
& \text { for } x_{2}>x_{2}^{0} \text { (or } x_{2}<x_{2}^{0} \text { ), it holds } \\
& \left.\operatorname{sgn}\left[P_{m}^{(2,2)}\left(x^{0}, \varphi_{x}\left(x^{0}\right)\right)\right] \cdot P_{m}\left(x, f_{x}\right)\right|_{\varnothing}>0 .
\end{aligned}
$$

Then we have the following uniqueness theorem.

Theorem. Under the assumptions (4) and (5), if $u \in \mathscr{D}^{\prime}(\Omega)$ is a solution of $P(x, \partial / \partial x) u=0$ and vanishes in $f(x)<f\left(x^{0}\right)$, then $u=0$ in a neighborhood of $x^{0}$.

Remark 1.1. The assumptions (4) and (5) mean the following: the double characteristic curve $\mathscr{S}$ and the characteristic curve in $\varphi(x)>\varphi\left(x^{0}\right)$ which is tangent at $x^{0}$ to $\mathscr{S}$ guaranteed its existence from the Proposition, lie in the domain where we require that $u=0$, when $x_{2}>x_{2}^{0}$ (or $x_{2}<x_{2}^{0}$ ) (see $\S 3$ ).

For the proof of the theorem we use the following lemma due to L. Hörmander $[5$, p. 125$]$.

Lemma. Let $P(x, \partial / \partial x)=\sum_{|\alpha| \leqq m} a_{\alpha}(x)(\partial / \partial x)^{\alpha}$ be a differential operator of order $m$ defined in an open set $\Omega \subset R^{n}$ with analytic coefficients, and 
assume that the coefficient of $\left(\partial / \partial x_{n}\right)^{m}$ does not vanish in $\Omega$. If $u \in \mathscr{D}^{\prime}(\Omega)$ is a solution of $P(x, \partial / \partial x) u=0$ in $\Omega_{c}=\left\{x ; x \in \Omega, x_{n}<c\right\}$ for some $c$, then $u=0$ in $\Omega_{c}$ provided $\Omega_{c} \cap \operatorname{supp}[u]$ is relatively compact in $\Omega_{\text {. }}$

Remark 1.2. We also obtain a uniqueness theorem if we assume that $f(x)>f\left(x^{0}\right)$ on $\mathscr{S}, x_{2}>x_{2}^{0}$ (or $\left.x_{2}<x_{2}^{0}\right)$ and $u=0$ in $f(x)>f\left(x^{0}\right)$. We omit the proof, since it is easier than that of Theorem.

\section{§2. Proof of Proposition}

Before the proof of our Proposition, we shall prove the following lemma.

Lemma 2.1. Let us consider the following algebraic equation in $z$,

$$
x_{1} z^{m}+x_{1} a_{1}(x) z^{m-1}+a_{2}(x) z^{m-2}+\cdots+a_{m}(x)=0,
$$

where $a_{i}(x)$ are real-valued analytic functions defined in a neighborhood of the origin $\Omega \subset R^{2}$. Assume that $a_{2}(x)<-\delta$ in $\Omega$ for some positive constant $\delta$, and let $z=z_{i}(x), i=1, \ldots, m$ be roots of (6) at $x_{1} \neq 0$. Then there exist two and only two real-valued analytic roots in $\left\{z_{i}(x)\right\}_{i=1}^{m}$, say $z_{i}(x), i=1,2$, in $\Omega_{+, \varepsilon}=\left\{x ; x \in \Omega, 0<x_{1}<\varepsilon\right\}$ such that

$$
z_{i}(x)=c_{i}(x) \cdot x_{1}^{-1 / 2} \quad \text { as } \quad x_{1} \downarrow 0,
$$

where $\varepsilon_{1}<\left|c_{i}(x)\right|<M$ for some positive constants $\varepsilon_{1}$ and $M_{9} c_{1}{ }^{\circ} c_{2}<0$ and $\varepsilon$ is a sufficiently small positive constant. And the other roots satisfy $\left|z_{i}(x)\right|$ $=O(1)$ uniformly in $x_{2}$ as $x_{1} \downarrow 0, i=3, \ldots, m$ in $\Omega_{+}=\left\{x ; x \in \Omega, x_{1}>0\right\}$. In $\Omega_{-}=\left\{x ; x \in \Omega, x_{1}<0\right\}$, all the real roots satisfy $z_{i}(x)=O(1)$ uniformly in $x_{2}$ as $x_{1} \uparrow 0$.

We prove the lemma by using the following classical result concerning algebraic equation.

Lemma. (Laguerre) Let us consider the following algebraic equation with real coefficients,

$$
f(z)=a_{0} z^{m}+a_{1} z^{m-1}+\cdots+a_{m}=0, \quad a_{0} \neq 0 .
$$


And now let $N$ be the number of roots which are greater than a given positive number $\alpha$, and $M$ be the number of variation of signs of

$$
f_{k}(\alpha)=a_{0} \alpha^{k}+a_{1} \alpha^{k-1}+\cdots+a_{k}, \quad k=0, \cdots, m .
$$

Then it follows that $N=M-2 l$, where $l \in\left\{0,1, \ldots,\left[\frac{m}{2}\right]\right\}$.

Proof of Lemma 2.1. Let $N$ be a sufficiently large positive constant satisfying

$$
N \geqq \max _{i \neq 2}\left\{\sup _{\Omega}\left|a_{i}(x)\right|\right\}, \quad \delta N^{2(k-2)}-\sum_{i=3}^{k} N^{2(k-i)+1}>\delta_{1},
$$

for $k=3, \cdots, m$, where $\delta_{1}$ is a positive constant less than $\delta$. Then we see that there exists one and only one real root $z=z_{1}(x)$ of (6) greater than $N^{2}$ in $\Omega_{+, \varepsilon}$, where $\varepsilon=\delta_{1} /\left(N^{2 m}+N^{2 m-1}\right)$ in view of the above lemma. In fact, let

$$
f_{k}(x, z)=x_{1} z^{k}+x_{1} a_{1}(x) z^{k-1}+a_{2}(x) z^{k-2}+\cdots+a_{k}(x),
$$

for $k=0,1, \ldots, m$, then we have that $f_{k}\left(x, N^{2}\right)>0, k=0,1$ and $f_{k}\left(x, N^{2}\right)$ $<0, k=2, \cdots, m$ in $\Omega_{+, \varepsilon}$. Since the number of variation of signs is one, there exists one and only one real root of (6) greater than $N^{2}$. Interchanging $z$ by $-z$ in (6), it also follows that there exists one and only one real root $z=z_{2}(x)$ of (6) smaller than $-N^{2}$ in $\Omega_{+, \varepsilon}$. In order to see that $z_{i}(x)=c_{i}(x) \cdot x_{1}^{-1 / 2}$ as $x_{1} \downarrow 0$, where $c_{1}>0$ and $c_{2}<0$, it sufficies to see that $\varepsilon_{1} / \sqrt{x_{1}}<z_{1}(x)<M / \sqrt{x_{1}}$ and $-M / \sqrt{x_{1}}<z_{2}(x)<-\varepsilon_{1} / \sqrt{x_{1}}$ if $x_{1}>0$ and $x_{1}$ is sufficiently small, where $\varepsilon_{1}$ and $M$ are constants satisfying $\varepsilon_{1}^{m}$ $+a_{2}(x) \varepsilon_{1}^{m-2}<0$ and $M^{m}+a_{2}(x) M^{m-2}>0$ in $\Omega$. The analyticities of them follow immediately from the implicit function theorem. And also it follows that the other roots in $\Omega_{+, \varepsilon}$ are smaller than $N^{2}$ in absolute value from Rouché's theorem. In $\Omega_{-}=\left\{x ; 0>x_{1}>-\frac{\delta_{1}}{N^{2 m}+N^{2 m-1}}\right\}$, all the real roots of (6) are smaller than $N^{2}$ in absolute value, in view of the above lemma. This completes the proof.

Proof of the Proposition. Let us transform the coordinates as follows, 


$$
y_{1}=\varphi(x)-\varphi\left(x^{0}\right), \quad y_{2}=\psi(x)-\psi\left(x^{0}\right)
$$

in a neighborhood of $x^{0}$, where $\phi(x)$ is a real-valued analytic function defined in $\Omega$ satisfying $\partial(\varphi, \psi) /\left.\partial\left(x_{1}, x_{2}\right)\right|_{x=x^{0}} \neq 0$. Let $\tilde{P}(y, \partial / \partial y)$ be a differential operator transformed by (7) defined in a neighborhood of the origin $\tilde{\Omega} \subset R^{2}$, then the coefficients of $\partial^{m} / \partial y_{1}^{m-k} \partial y_{2}^{k}, k=0,1,2$ of $\tilde{P}(y$, $\partial / \partial y)$ are

$$
P_{m}\left(x, \varphi_{x}\right), \sum_{i=1}^{2} P_{m}^{(i)}\left(x, \varphi_{x}\right) \psi_{x_{i}} \text { and } \frac{1}{2} \sum_{i, j} P_{m}^{(i, j)}\left(x, \varphi_{x}\right) \psi_{x_{i}} \psi_{x_{j}}
$$

respectively. We note at first that we can represent $P_{m}\left(x(y), \varphi_{x}(x(y))\right)$ $=y_{1} \tilde{a}_{0}(y)$ since $P_{m}\left(x(y), \varphi_{x}(x(y))\right)=0$ when $y_{1}=0$, and we have easily

$$
\tilde{a}_{0}\left(0, y_{2}\right)=\left.\left[\frac{1}{\left|\varphi_{x}\right|^{2}}<\varphi_{x}, \frac{\partial}{\partial x}>P_{m}\left(x, \varphi_{x}\right)\right]\right|_{y_{1}=0}
$$

And also we can represent $\sum_{i} P_{m}^{(i)}\left(x(y), \varphi_{x}(x(y))\right) \psi_{x_{i}}(x(y))=y_{1} \tilde{a}_{1}(y)$, since $P_{m}^{(i)}\left(x(y), \varphi_{x}(x(y))\right)=0$ when $y_{1}=0$. Thus the characteristic polynomial $\tilde{P}_{m}(y, \eta)$ of $\tilde{P}(y, \partial / \partial y)$ is represented as follows,

$$
\begin{aligned}
& \tilde{P}_{m}(y, \eta)=y_{1} \tilde{a}_{0}(y) \eta_{1}^{m}+y_{1} \tilde{a}_{1}(y) \eta_{1}^{m-1} \eta_{2}+ \\
& +\tilde{a}_{2}(y) \eta_{1}^{m-2} \eta_{2}^{2}+\sum_{k=3}^{m} \tilde{a}_{k}(y) \eta_{1}^{m-k} \eta_{2}^{k},
\end{aligned}
$$

where $\tilde{a}_{2}(y)=\frac{1}{2} \sum_{i, j} P_{m}^{(i, j)}\left(x, \varphi_{x}\right) \psi_{x_{i}} \psi_{x_{j}}$.

Now we remark that if a curve defined by $g(y)=c,\left(g_{y_{2}} \neq 0\right)$ is a characteristic curve of $\tilde{P}(y, \partial / \partial y)$, it must satisfy $\tilde{P}_{m}\left(y, g_{y}\right)=0$. And also it follows that $g(y)=c$ is a solution of the following ordinary differential equation,

$$
\begin{aligned}
F\left(y, d y_{2} / d y_{1}\right) \equiv & y_{1} \tilde{a}_{0}(y)\left(-\frac{d y_{2}}{d y_{1}}\right)^{m}+y_{1} \tilde{a}_{1}(y)\left(-\frac{d y_{2}}{d y_{1}}\right)^{m-1}+ \\
& +\tilde{a}_{2}(y)\left(-\frac{d y_{2}}{d y_{1}}\right)^{m-2}+\sum_{k=3}^{m} \tilde{a}_{k}(y)\left(-\frac{d y_{2}}{d y_{1}}\right)^{m-k}=0
\end{aligned}
$$

in view of $-d y_{2} / d y_{1}=g_{y_{1}} / g_{y_{2}}$ on $g(y)=c$. Conversely it is obvious 
that the solutions of (8) are also characteristic curves of $\tilde{P}(y, \partial / \partial y)$. Let us consider the ordinary differential equation (8) instead of considering the partial differential equation $\tilde{P}_{m}\left(y, g_{y}\right)=0$.

In view of the assumptions that $\tilde{a}_{0}\left(0, y_{2}\right) \neq 0$, and $\operatorname{sgn}\left[\tilde{a}_{0}(0)\right]$ and $\operatorname{sgn}\left[\tilde{a}_{2}(0)\right]$ are different, we may assume

$$
\tilde{a}_{2}(y) / \tilde{a}_{0}(y)<-\delta
$$

in $\tilde{\Omega}$ for some positive constant $\delta$. From Lemma 2.1, there exist two and only two real-valued analytic roots of (8) with respect to $d y_{2} / d y_{1}$ in $\tilde{\Omega}_{+, \varepsilon}$ for a sufficiently small $\varepsilon$, say $d y_{2} / d y_{1}=f_{i}(y), i=1,2$, such that $f_{i}=c_{i}(y)$ $\cdot y_{1}^{-1 / 2}$ as $y_{1} \downarrow 0$, where $\varepsilon_{1}<\left|c_{i}(y)\right|<M$ for some positive constants $\varepsilon_{1}$ and $M$, and $c_{1} \cdot c_{2}<0$. Let us consider the following ordinary differential equations in $\widetilde{\Omega}_{+, \varepsilon}^{a}$,

$$
\frac{d y_{1}}{d y_{2}}=f_{i}^{-1}(y), \quad i=1,2
$$

where $\tilde{\Omega}_{+, \varepsilon}^{a}$ is a closure of $\tilde{\Omega}_{+, \varepsilon}$. Obviously the solutions of (9) are also characteristic curves of $\tilde{P}(y, \partial / \partial y)$. From the theory of ordinary differential equation, it follows that every solution of (9) is tangent to $y_{1}=0$ in view of $f_{i}^{-1}(y)=c_{i}^{-1}(y) \cdot y_{1}^{1 / 2}$ as $y_{1} \downarrow 0$. In order to see that they are tangent at the second order to $y_{1}=0$, it suffices to show $\lim _{y_{1} \downarrow 0} d^{2} y_{1} / d y_{2}^{2} \neq 0$. Now we use the following relation,

$$
d^{2} y_{1} / d y_{2}^{2}=-\left[\left(f_{i}\right)_{y_{1}} / f_{i}^{3}+\left(f_{i}\right)_{y_{2}} / f_{i}^{2}\right]
$$

Since $f_{i}(y)$ satisfies $F\left(y, f_{i}\right) \equiv 0$ in $\widetilde{\Omega}_{+, \varepsilon}$, we have

$$
\begin{aligned}
& \left(f_{i}\right)_{y_{1}}=-F_{y_{1}}\left(y, f_{i}\right) / F_{z}\left(y, f_{i}\right), \\
& \left(f_{i}\right)_{y_{2}}=-F_{y_{2}}\left(y, f_{i}\right) / F_{z}\left(y, f_{i}\right),
\end{aligned}
$$

where $F_{y_{k}}(y, z)=\partial F(y, z) / \partial y_{k}, k=1,2$ and $F_{z}(y, z)=\partial F(y, z) / \partial z$. Now we shall consider $F_{z}\left(y, f_{i}\right)$ and $F_{y_{k}}\left(y, f_{i}\right), k=1,2$; 


$$
\begin{gathered}
-F_{z}\left(y, f_{i}\right)=m y_{1} \tilde{a}_{0}\left(-f_{i}\right)^{m-1}+(m-1) y_{1} \tilde{a}_{1}\left(-f_{i}\right)^{m-2} \\
+(m-2) \tilde{a}_{2}\left(-f_{i}\right)^{m-3}+\sum_{k=3}^{m-1}(m-k) \tilde{a}_{k}\left(-f_{i}\right)^{m-k-1}, \\
F_{y_{1}}\left(y, f_{i}\right)=\tilde{a}_{0}\left(-f_{i}\right)^{m}+y_{1}\left(\tilde{a}_{0}\right)_{y_{1}}\left(-f_{i}\right)^{m}+\left(y_{1} \bullet \tilde{a}_{1}\right)_{y_{1}}\left(-f_{i}\right)^{m-1} \\
+\sum_{k=2}^{m}\left(\tilde{a}_{k}\right)_{y_{1}}\left(-f_{i}\right)^{m-k}, \\
F_{y_{2}}\left(y, f_{i}\right)=y_{1}\left(\tilde{a}_{0}\right)_{y_{2}}\left(-f_{i}\right)^{m}+y_{1}\left(\tilde{a}_{1}\right)_{y_{2}}\left(-f_{i}\right)^{m-1} \\
+\left(\tilde{a}_{2}\right)_{y_{2}}\left(-f_{i}\right)^{m-2}+\sum_{k=3}^{m}\left(\tilde{a}_{k}\right)_{y_{2}}\left(-f_{i}\right)^{m-k} .
\end{gathered}
$$

Considering $f_{i}(y)=c_{i}(y) y_{1}^{-1 / 2}$ as $y_{1} \downarrow 0$, we have

$$
F_{z}\left(y, f_{i}\right)=d_{i}\left(y_{2}\right) y_{1}^{-(m-3) / 2}+o\left(y_{1}^{-(m-3) / 2}\right) \text { as } y_{1} \downarrow 0 \text {, }
$$

where $d_{i}\left(y_{2}\right) \neq 0$. And also we have

$$
\begin{aligned}
& F_{y_{1}}\left(y, f_{i}\right)=e_{i}\left(y_{2}\right) y_{1}^{-m / 2}+o\left(y_{1}^{-m / 2}\right) \text { as } y_{1} \downarrow 0, \\
& F_{y_{2}}\left(y, f_{i}\right)=O\left(y_{1}^{-(m-2) / 2}\right) \text { as } y_{1} \downarrow 0,
\end{aligned}
$$

where $e_{i}\left(y_{2}\right) \neq 0$. Thus we have

$$
\begin{gathered}
\lim _{y_{1} \downarrow 0}\left(f_{i}\right)_{y_{1}} / f_{i}^{3} \neq 0, \\
\left(f_{i}\right)_{y_{2}} / f_{i}^{2}=O\left(y_{1}^{1 / 2}\right) \quad \text { as } y_{1} \downarrow 0 .
\end{gathered}
$$

Therefore the solutions of (9) are tangent at the second order to $y_{1}=0$. Finally, it is obvious that the other characteristic curves of $\tilde{P}(y, \partial / \partial y)$ are transversal to $y_{1}=0$, because the other real roots of the algebraic equation $F(y, z)=0$ in $z$ at $y_{1} \neq 0$ are $O(1)$ as $y_{1} \downarrow 0$ or $y_{1} \uparrow 0$ (Lemma 2.1). This completes the proof.

\section{§3. Proof of Theorem}

It suffices to prove our Theorem for the partial differential operator $\tilde{P}(y, \partial / \partial y)$ in $\tilde{\Omega}$ considered in the previous section. Let $\tilde{P}(y, \partial / \partial y)$ be 


$$
\tilde{P}(y, \partial / \partial y)=\tilde{P}_{m}(y, \partial / \partial y)+Q(y, \partial / \partial y)
$$

where $\tilde{P}_{m}(y, \partial / \partial y)=y_{1} \tilde{a}_{0}(y) \frac{\partial^{m}}{\partial y_{1}^{m}}+y_{1} \tilde{a}_{1}(y) \frac{\partial^{m}}{\partial y_{1}^{m-1} \partial y_{2}}+\tilde{a}_{2}(y) \frac{\partial^{m}}{\partial y_{1}^{m-2} \partial y_{2}^{2}}+$ $+\sum_{k \geqq 3} \tilde{a}_{k}(y) \frac{\partial^{m}}{\partial y_{1}^{m-k} \partial y_{2}^{k}}, \tilde{a}_{0}(y) \cdot \tilde{a}_{2}(y)<0$ in $\tilde{\Omega}$, and $Q(y, \partial / \partial y)$ is a differential operator of order $m-1$.

Let $\tilde{\mathscr{C}}$ be an image of $\mathscr{C}$ by the transformation (7), and set

$$
\tilde{\mathscr{C}}: \tilde{f}(y)=\tilde{f}(0),\left(\left(\tilde{f}_{y_{1}}(0), \tilde{f}_{y_{2}}(0)\right)=(1,0), \tilde{f}(y) \in C^{1}((\tilde{\Omega})) .\right.
$$

Then the conditions of Theorem become as follows,

$$
\begin{aligned}
& \tilde{f}\left(0, y_{2}\right)<\tilde{f}(0), y_{2}>0 \quad\left(\text { or } y_{2}<0\right), \\
& \left.\operatorname{sgn}\left[\tilde{a}_{2}(0)\right] \cdot \tilde{P}_{m}\left(y, \tilde{f}_{y}\right)\right|_{\tilde{E}}>0, y_{2}>0 \quad\left(\text { or } y_{2}<0\right) .
\end{aligned}
$$

At first we note the meaning of these conditions. From the Proposition, there exist two and only two characteristic curves through each point in $\widetilde{\Omega}_{+, \varepsilon}$ which are tangent at the second order to $y_{1}=0$, and they are represented by the solutions of the ordinary differential equations (9). Without loss of generality, we may assume that $f_{1}^{-1}>0$ and $f_{2}^{-1}<0$ in $\tilde{\boldsymbol{\Omega}}_{+, \varepsilon}$. Then it follows immediately from (11) that

$$
\begin{aligned}
& -\tilde{f}_{y_{2}} / \tilde{f}_{y_{1}}>f_{1}^{-1} \quad \text { on } \tilde{\mathscr{C}}, y_{2}>0, \\
& \left(\text { or }-\tilde{f}_{y_{2}} / \tilde{f}_{y_{1}}<f_{2}^{-1} \text { on } \tilde{\mathscr{C}}, y_{2}<0\right) .
\end{aligned}
$$

These have a geometrical meaning as stated in Remark 1.1 in $\$ 1$.

We shall prove the theorem only in the case where $-\tilde{f}_{y_{2}} / \tilde{f}_{y_{1}}>f_{1}^{-1}$ on $\tilde{\mathscr{C}}, y_{2}>0$, since the proof is the same in the other case. We may assume that $f_{1}^{-1}(y)$ is monotonically increasing with respect to $y_{1}$ in $\tilde{\Omega}_{+, \varepsilon}$, because of $f_{1}^{-1}(y)=c_{1}^{-1}(y) \cdot y_{1}^{1 / 2}$ as $y_{1} \downarrow 0$. Now let us consider the following ordinary differential equation,

$$
\frac{d y_{1}}{d y_{2}}=f_{1}^{-1}\left(y_{1}+\alpha, y_{2}\right) \text { in } \widetilde{\Omega}_{\alpha},
$$

where $\alpha$ is a sufficiently small positive constant $(\alpha<\varepsilon)$ and $\tilde{\Omega}_{\alpha}$ is defined 
by $\tilde{\Omega}_{\alpha}=\left\{y ;\left(y_{1}+\alpha, y_{2}\right) \in \tilde{\Omega}_{+, \varepsilon}, y_{1}>-\alpha / 2\right\}$. Since the right hand of (13) is analytic in the domain $\tilde{\Omega}_{\alpha}$, (13) has not any singular solution, and the solutions of (13) are not characteristic curves of $\tilde{P}(y, \partial / \partial y)$ in $\tilde{\Omega}_{\alpha}$, because of the assumptions that $f_{1}^{-1}(y)$ is monotonically increasing with respect to $y_{1}$ and the other characteristic curves are transversal to $y_{1}=0$. And now let

$$
\tilde{\varphi}_{\alpha}(y)=c \quad \text { in } \tilde{\Omega}_{\alpha}
$$

be solutions of (13), then in view of (12) it follows that the domain enclosed by $\tilde{\mathscr{C}}$ and $\tilde{\varphi}_{\alpha}(y)=\tilde{\varphi}_{\alpha}(0)$ is relatively compact in $\tilde{\Omega}_{\alpha}$ if we choose $\alpha$ sufficiently small. In fact, $\tilde{\varphi}_{\alpha}(y)=\tilde{\varphi}_{\alpha}(0)$ is sufficiently near to the solution of $d y_{1} / d y_{2}=f_{1}^{-1}(y)$ which is tangent at the origin to $y_{1}=0$ if $\alpha$ is small. Thus we see that

$$
\left\{y ; \tilde{\varphi}_{\alpha}(y)=\tilde{\varphi}_{\alpha}\left(0, y_{2}\right), y_{2}>-\delta\right\} \cap \operatorname{supp}[u]
$$

is relatively compact in $\tilde{\Omega}_{\alpha}$ if we choose a sufficiently small positive constant $\delta$, since $u=0$ in $\tilde{\Omega}_{\alpha} \cap\{y ; \tilde{f}(y)<\tilde{f}(0)\}$.

Now let us transform the coordinates in $\tilde{\Omega}_{\alpha}$ as follows,

$$
z_{1}=\tilde{\varphi}_{\alpha}(y), \quad z_{2}=y_{2} \text {. }
$$

Then it is obvious that $\partial\left(z_{1}, z_{2}\right) / \partial\left(y_{1}, y_{2}\right) \neq 0$ in $\tilde{\Omega}_{\alpha}$. Let $\tilde{P}(z, \partial / \partial z)$ be a partial differential operator transformed by the above transformation. Then the coefficient of $\left(\partial / \partial z_{1}\right)^{m}$ of $\tilde{\widetilde{P}}(z, \partial / \partial z)$ does not vanish in the domain considered, since $z_{1}=$ const. is not characteristic. And obviously the assumption of Lemma in $\S 1$ is satisfied for the equation $\tilde{\widetilde{P}}(z, \partial / \partial z) u$ $=0$, therefore $u=0$ in $\left\{z ; z_{1}=\tilde{\varphi}_{\alpha}\left(0, y_{2}\right), y_{2}>-\delta\right\}$, that is, $u=0$ in $\left\{y \in \tilde{\Omega}_{\alpha} ; \tilde{\varphi}_{\alpha}(y)=\tilde{\varphi}_{\alpha}\left(0, y_{2}\right), y_{2}>-\delta\right\}$. This completes the proof.

\section{References}

[1] Bony, J.M., Sur la propagation des maximums et l'unicité du problème de Cauchy pour les opérateurs elliptiques dégénérés du second ordre, C.R. Acad. Sci. Paris, 266 (1968), 763-765.

[2] Une extension du théorème de Holmgren sur l'unicité de problème de Cauchy, C.R. Acad. Sci. Paris, 268 (1969), 1103-1106. 
[3] Hasegawa, Y., On the initial-value problem with data on a double characteristic, J. Math. Kyoto Univ. 11 (1971), 357-372.

[4] — On the initial-value problem with data on a characteristic plane, to appear.

[5] Hörmander, L., Linear Partial Differential Operators, Springer.

[6] - Uniqueness theorems and wave front sets for solutions of linear differential equations with analytic coefficients, Comm. Pure Appl. Math. 24 (1971), 671-704.

[7] A remark on Holmgren's uniqueness theorem, J. Diff. Geom. 6 (1971), 129-134.

[8] Miyake, M., On the initial-value problems with data on a characteristic surface for linear systems of first order equations, Publ. R.I.M.S. Kyoto Univ. 8 (1972/73), 231-264.

[9] Trèves, F., Linear Partial Differential Equations with Constant Coefficients, Gordon and Breach, New York, 1966.

[10] Vaillant, J., Données de Cauchy portées par une caractéristique double..., $J$. Math. Pures Appl. 47 (1968), 1-40.

[11] Zachmanoglou, E.C., Uniqueness of the Cauchy problem for linear partial differential equations, Trans. Amer. Math. Soc. 136 (1969), 517-526.

[12] - Propagation of zeros and uniqueness in the Cauchy problem for first order partial differential equations, Arch. Rat. Mech. Anal. 38 (1970), 178-188. 
